\title{
A WALKING ROBOT WITH THERMOMECHANICAL ACTUATORS FOR THE INSPECTION OF PHOTO-ELECTRIC CELLS OF SOLAR ARRAYS FOR SPACECRAFT
}

\author{
ANDREI ZHUKOV \\ Moscow Aviation Institute (National Research University), \\ Volokolamskoe Shosse, Moscow, 125993, Russia \\ E-mail: and_zhukov@mail.ru \\ NIKOLAY BOLOTNIK and VLADISLAV CHASHCHUKHIN \\ Laboratory of Robotics and Mechatronics, \\ Institute for Problems in Mechanics of the Russian Academy of Sciences, \\ 101-1 Vernadsky Ave, Moscow, 119526, Russia \\ E-mail: bolotnik@ipmnet.ru,ketlk@mail.ru
}

\begin{abstract}
The paper discusses a concept of using a walking robot with thermomechanical actuators and adhesive contact devices for the inspection of the technical state of the cells of solar arrays on space stations.
\end{abstract}

\section{Introduction}

At the present time, the technical state of the solar arrays on International Space Station (ISS) is monitored by sensors. If the readings of the sensors change gradually or sharply, the array should be inspected for failures. Inspection works are produced by astronauts outside the station [1], which increases the life and health risk for the astronauts. The ISS solar array has an overall area of $2400 \mathrm{~m}^{2}$ and consists of four modules [2], of an area of $600 \mathrm{~m}^{2}$ each. The inspection of these modules is a time-consuming and labor-consuming task.

In our paper, we discuss the possibilities for the inspection of ISS solar arrays by means of a space robot. To develop such a robot one should

- analyze the structure of the solar array and the factors that deteriorate its operating parameters;

- analyze the inspection techniques available for solar arrays;

- analyze the achievements in mini-robotics, in particular, in space robotics;

- propose a design schematic of a mini-robot and analyze its locomotion principles and possibilities;

- perform a feasibility analysis of the proposed engineering solution.

\section{ISS solar arrays and the factors that deteriorate their operating parameters}

ISS is equipped with framed solar arrays. They are assembled from photoelectric transducers (photoelectric cells) on a carrying frame $[2,3]$.

The photoelectric cells are implemented as silicon plates with current conducting buses and diodes; the cells are coated with protecting glasses on both sides.

The solar arrays can be subjected to mechanical impacts of micro-meteorites, as a result of which "craters" appear on the array surface; these craters are randomly distributed over the surface. For example, on the panel of the solar array that has been employed for 10 
years on MIR station and has been brought back on earth, from 5 to 15 holes of 1 to $2 \mathrm{~mm}$ in diameter were detected in any direction per each meter of length (Fig. 1) [2].

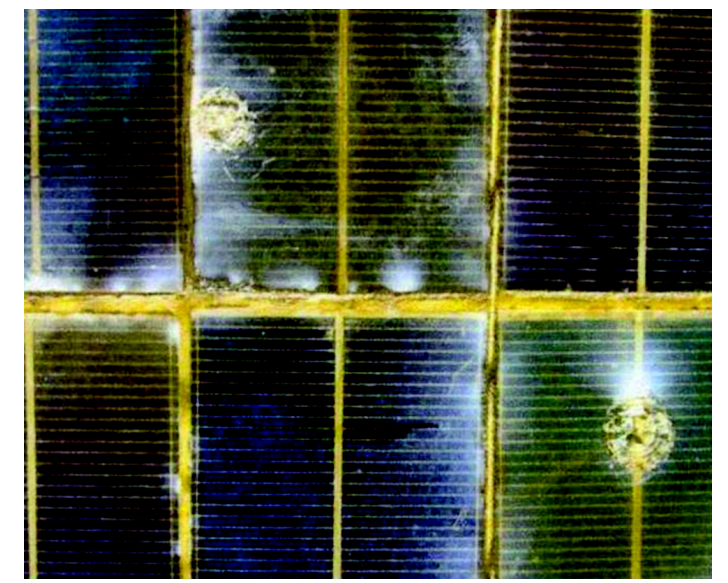

Fig. 1. Typical micro-meteorite holes on a panel of the solar array of MIR space station.

\section{Available and prospective techniques for the inspection of solar arrays}

At the present time, the inspection is performed either remotely by means of the current sensors integrated in the array or visually by astronauts. In the remote inspection, a failure is detected by changes in the total current produced by all generators. The value of this current is refined according to the readings of the consumed current sensors and is compared with the sum of the currents produced by each of the generators.

A prospective way to automate the inspection of the solar arrays and to increase the inspection reliability is to use mobile space robots.

The motion of a micro-robot along the surface of a spacecraft solar array can be enabled by two different physical mechanisms that are characterized by different kinematics. Each of these mechanisms needs a specific hardware implementation.

Adhesion / detachment mechanism is based on the intermolecular attraction of a dry adhesive to a surface that has micro-roughness and contains micro-particles settled down on it. The foot of the robot is connected to its leg by a controlled thermomechanical revolute joint. When adhering (Fig. 2), the contact surface of the foot is parallel to the underlying surface. To detach the foot from the surface, a control torque is applied to the joint, and the foot detaches from the surface with formation of a crack that propagates along the interface. The gradual detachment through a crack requires significantly less force from the actuator than the simultaneous detachment of the entire contact surface.

Grasping mechanism. To use this mechanism, the robot should have grasping devices on the extremities that are controlled according to a prescribed algorithm. Having grasped the solar array surface with the front extremities, the robot pulls itself forward and grasps the surface with the rear extremities thus moving along.

Both suggested principles of motion have advantages and drawbacks, which should be taken into account when designing a robot for specific operating conditions. The grasping devices require the robot to be positioned on the cells of the spacecraft solar arrays with high accuracy. The adhesion / detachment mechanism fails to work if the contact surface of the robot's foot is contaminated with micro-particles; in this case, the robot loses its ability to move. 


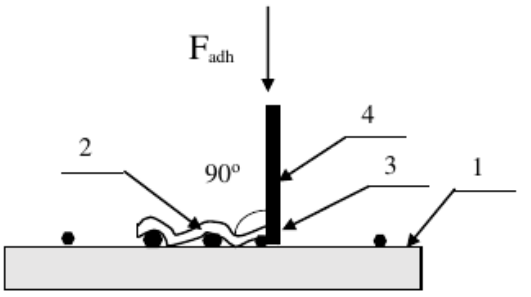

a)

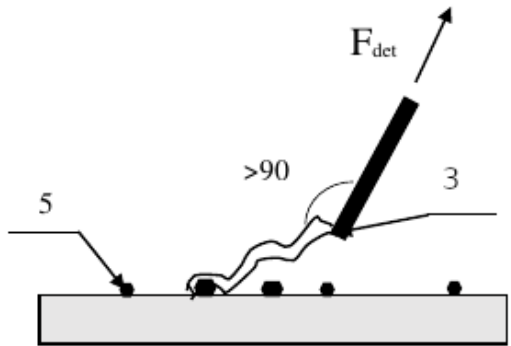

b)

Fig. 2. Adhesion (a) / detachment (b) cycle for the extremity of the micro-robot. 1: underlying surface, 2: foot of the robot, 3: controlled joint of the robot's foot, 4: leg of the robot, 5: micro-particles settled down on the underlying surface.

A new mobile space robot is under development in Russia [4] with a prospect to be delivered to ISS in 2021. This robot will be used for the extravehicular maintenance of spacecraft and for assisting astronauts. Using this robot for the inspection of solar arrays will require building rigging structures, basing points, and handrails on the array, which will decrease the inspection efficiency.

An alternative engineering solution can be based on using walking mechanisms. A four-limbed robot LEMUR (Limited Excursion Mechanical Utility Robot) was designed at NASA's Jet Propulsion Laboratory (Pasadena, California, USA) [5]. This robot can climb rock walls, gripping with multiple tiny hooks in each of its fingers. It has an artificial intelligence to find its way around obstacles.

In [6], the tasks that can be implemented by walking mini-robots in space are formulated and the respective requirements that are imposed on the structural and operating parameters of such robots are discussed. A concept of a walking space mini-robot with thermomechanical silicon-polyimide actuators is proposed. When operating in microgravity conditions, the feet of the walking mechanisms must adhere to and detach from an underlying surface in a controlled mode.

In [7], the creation of a micro-robot HAMR-E (Harvard Ambulatory Micro-Robot with Electroadhesion) is reported. The robot has a length of $4.5 \mathrm{~cm}$ and can move along vertical surfaces due to electroadhesion. The robot has a mass of $1.48 \mathrm{~g}$, and a voltage of $250 \mathrm{~V}$ is needed to provide adhesion. The robot can move along vertical surfaces at a velocity of $1.2 \mathrm{~mm} / \mathrm{s}$. However, electroadhesive devices require a significant voltage that can have a negative influence on the operation of the photoelectric transducers. This robot was tested in the conditions of earth gravity and its behavior in the microgravity conditions has not been investigated.

A wall-climbing micro-robot that uses dry adhesion is considered in [8]. This type of adhesion is accounted for by Van der Waals forces that act between the fibrillar structures of the robot's feet and the underlying surface. This type of adhesion may appear suitable for the microgravity conditions. To apply such types of walking robots for inspecting solar arrays one should take into account that products of the proper external atmosphere of the space station (e.g, the products of the sputtering of the materials of the engine jets) can condense on the surface of the solar array [9].

Therefore, for creating a walking mechanism that can operate under microgravity, it is necessary to solve a scientific and engineering problem of controlled adhesion/detachment interaction of the robot with the surfaces of the photoelectric transducers based on dry adhesives. Thermomechanical actuators are proposed to be used as the propelling devices. Such actuators are proved to be suitable for operating outside a spacecraft. It is reason- 
able that the dimensions of the robot be commensurable with the dimensions of a single photoelectric transducer. Proceeding from the purpose of the robot and the conditions of operation in space, we can formulate technical requirements to be imposed on the robot. These requirements are summarized in Table below.

\begin{tabular}{|c|c|c|}
\hline Operating characteristic & Value & Comment \\
\hline Maximum step length, mm & 80 & $\begin{array}{l}\text { Walking along the longer side } \\
\text { of the photoelectric cell. }\end{array}$ \\
\hline Minimum step length, mm & 50 & $\begin{array}{l}\text { Walking along the shorter } \\
\text { side of the photoelectric cell. }\end{array}$ \\
\hline $\begin{array}{l}\text { Number of mini-robots per } \\
\text { each module }\end{array}$ & 4 (2 for each array) & $\begin{array}{l}\text { Inspection of each module of } \\
\text { the ISS solar array. }\end{array}$ \\
\hline $\begin{array}{l}\text { Maximum dimension of the } \\
\text { open gripper, } \mathrm{mm}\end{array}$ & $0.1 \ldots 0.5$ & Motion with gripping. \\
\hline Power reserve, $\mathrm{m}$ & 10000 & $\begin{array}{l}\text { For one module of the array } \\
\left(600 \mathrm{~m}^{2}\right) .\end{array}$ \\
\hline Resource, million cycles & 20 & $\begin{array}{l}\text { Allows the robot to move af- } \\
\text { ter recharging the batteries. }\end{array}$ \\
\hline Radiation strength, krad & 100 & $\begin{array}{l}\text { Corresponds to the operating } \\
\text { conditions in space. }\end{array}$ \\
\hline Operating temperature, ${ }^{\circ} \mathrm{C}$ & $-150 \ldots+150$ & $\begin{array}{l}\text { Corresponds to the operating } \\
\text { conditions in space. }\end{array}$ \\
\hline Average velocity, mm/min & 50 & $\begin{array}{l}\text { Photoelectric array inspec- } \\
\text { tion. }\end{array}$ \\
\hline Power supply voltage, $\mathrm{V}$ & 5 & $\begin{array}{l}\text { Power supply for the motion, } \\
\text { control, camera, and memory. }\end{array}$ \\
\hline Camera resolution, dpi & 300 & For obtaining micro-photos. \\
\hline SD card volume, GB & 600 & Mapping of the array surface. \\
\hline
\end{tabular}

\section{Design of the robot with thermomechanical actuators}

The robot consists of a plate on which thermomechanical actuators that play at the same time the role of propelling devices are arranged. The module of the robot that involves four such actuators is schematically shown in Fig. 3. Each actuator may contain an additional segment that plays the role of a foot and serves for increasing the reliability of the adhesive contact of the robot with the underlying surface. The actuators are arranged pairwise along one line; in each pair the actuators are turned relative one another by an angle of $180^{\circ}$. The flow chart of the motion of the actuators arranged in such a way is shown in Fig. 3 . The robot has two pairs of modules. One pair implements the motions along the shorter sides of the photoelectric transducers, while the other pair provides the motions along the longer sides (Fig. 4). The robot may adjust the direction of its motion due to small rotation of its body. The rotation is performed if only the legs of one side are active, while the legs of the other side are fixed on the underlying surface. The rotation is possible due to elastic bending of the legs that are fixed to the surface. The approach of the robot to an edge of the photoelectric transducer is detected by the edge sensors that can be based on optical pairs. The inspection of the surface of the solar array is produced by processing the 


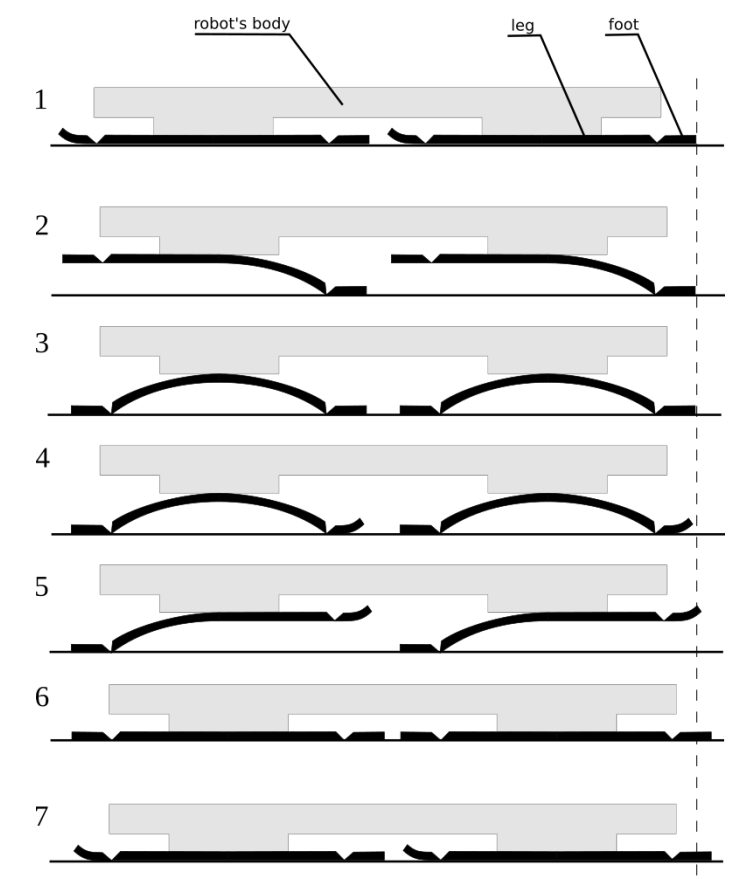

Fig. 3. Flow chart of the gait of the robot for one step.

sequence of the images obtained from a camera that is installed on board of the robot and photographs the area shown in Fig. 4. The position and the orientation of the robot are identified by processing the data obtained from a video camera. Possibly, for this purpose one would have to use an additional camera with lower resolution to save computational resources of the on-board micro-controller. On the images obtained from the navigation camera, regions that correspond to the junctions between separate cells of the photoelectric arrays are distinguished. The control signals applied to the actuators should be coordinated to provide the motion of the robot along the junction line of the cells of the photoelectric arrays.

\section{An algorithm for inspection of the solar array by the walking robot}

The algorithm provides scanning the surface of a solar array along the trajectory shown in Fig. 4. The robot attaches itself to the surface of the solar array by means of limbs with dry adhesives. The position and the orientation of the robot are identified by means of the edge sensors and a video camera.

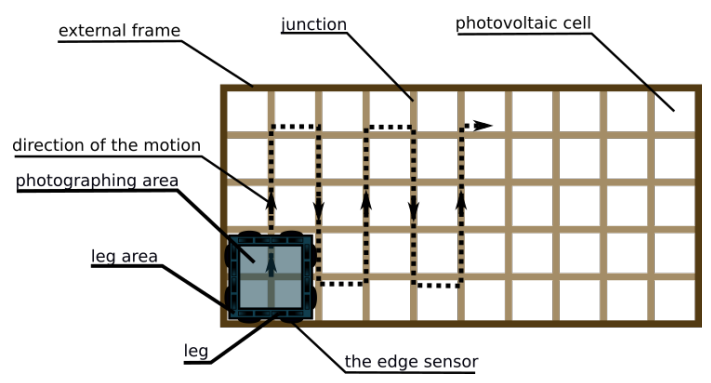

Fig. 4. The motion of the robot along the solar array. 


\section{Conclusions}

A concept of a walking robot that enables nondestructive inspection of the photoelectric cells of solar arrays of spacecraft is proposed. The technical requirements for the robot are presented. The design schematic of the robot is suggested and the equipment necessary for its functioning is defined. An algorithm of the inspection of the solar array surface by means of the walking robot with thermomechanical actuators is described.

\section{Acknowledgments}

This research is partly supported by Program \# 7 "New Developments in Prospective Areas of Power Engineering, Mechanics, and Robotics" of the Presidium of the Russian Academy of Sciences.

\section{References}

1. Zernov, A.S., Nikolaev, V.D.: Experience of operating solar arrays on the service module of the International Space Station. Kosmicheskaya Tekhnika i Tekhnologii (Space Engineering and Technologies), no. 2, 29-38 2016. [in Russian]

2. Melnikov, V.M., Matyushenko, I.N., Chernova, N.A., Kharlov, B.N.: Problems of creation of large-scale structures in space. Trudy MAI (Proceedings of Moscow Aviation Institute), no 78 (2014). [in Russian]

3. http//npp-kvant.ru

4. Kosmorobot: Mobole robot for operations outside a spacecraft. http://rtc.ru/solution/ kosmorobot [in Russian]

5. New Commercial Robot Copies Geckos' Toes: https://www.nasa.gov/directorates/ spacetech/spinoff/New_Commercial_Robot_Copies_Geckos_Toes (2020)

6. Bolotnik, N.N., Gradetskii V.G., Zhukov A.A., et al.: Mobile Space Microrobot: Concept and Application Prospects. Cosmic Research, 57(2), 115-120 (2019).

7. De Rivaz, S.D., Goldberg, B.,Doshi, N., Jayaram, K.: Inverted and vertical climbing of a guadrupedal microrobot using electroadhesion. Sci. Robot. 3 (25) eaau 3038 (2018).

8. Greuter, G., Shan, G., Caprari, G., Tache, F., Siegwart, R., Sitti,, M.: Toward micro wallclimbing robots using biomimetic fibrillar adhesives.

9. Akishin, A.I.: Impact of the spacecraft's own external atmosphere on their materials and equipment. Perspektivnye Materialy (Advanced Materials), no. 2, 14-22 (2007). [in Russian] 